\title{
OCORRÊNCIA DE Psyllaephagus bliteus RIEK (HYMENOPTERA: ENCYRTIDAE) NO BRASIL
}

\author{
Evoneo Berti Filho ${ }^{1}$ \\ Valmir Antonio Costa ${ }^{2}$ \\ Robert L. Zuparko ${ }^{3}$ \\ John LaSalle ${ }^{4}$
}

Psyllaephagus é um gênero com algumas espécies de importância no controle biológico de psilídeos que atacam mirtáceas (Myrtaceae). Na Califórnia, EUA, foram introduzidas da Austrália as espécies: Psyllaephagus pillosus Riek (Encyrtidae), para o controle de Ctenarytaina eucalipti (MaskelI) (Hemiptera: Psyllidae) em Eucalyptus pulverulenta Sims, e Psyllaephagus bliteus Riek (Encyrtidae), para o controle de Glycaspis brimblecombei (Hemiptera: Psyllidae), em Eucalyptus camaldulensis. No Brasil, a primeira espécie do gênero Psyllaephagus, P. baccharidis sp.n., foi registrada parasitando ninfas de Neopelma baccharidis Burckhardt (Hemiptera: Psyllidae) em carqueja (Baccharis dracunculifolia) em São Carlos e Ribeirão Bonito SP (Tavares \& Perioto, 1993) e Psyllaephagus sp. (Hymenoptera: Encyrtidae) foi encontrado parasitando Triozoida sp. (Hemiptera: Psyllidae) atacando brotações de goiabeira (Psidium guajava) em Campinas e Valinhos, SP (V.A.Costa,comunicação pessoal). O psilídeo-de-concha, Glycaspis brimblecombei (Hemiptera: Psyllidae), foi recentemente detectado no Brasil em Eucalyptus camaldulensis e E. tereticornis. (C.F.Wilcken, Alerta PROTEF, 2003). Na Fazenda Areão, da Escola Superior de Agricultura Luiz de Queiroz (ESALQ/USP) em Piracicaba, SP, foram coletados galhos e folhas de Eucalyptus camaldulensis e E. tereticornis atacados pelo psilídeo-de-concha, G brimblecombei, e mantidos em gaiolas no Laboratório de Entomologia Florestal da ESALQ/USP. Observou-se a emergência de um grande número de himenópteros que foram identificados pelos Drs. J. LaSalle (Austrália) e R. L. Zuparko (Califórnia, EUA), como Psyllaephagus bliteus/quadricyclus (sinonímia não publicada). Na Austrália, Riek (1962) registrou P.bliteus parasitando Glycaspis sp. em Eucalyptus blakely, E.camaldulensis e E.rossii e P.quadricyclus parasitando Glycaspis sp.em Eucalyptus blakely, E.melliodora, E.rossii e E.viminalis. Este é o primeiro registro da ocorrência de Psyllaephagus bliteus/quadricyclus no Brasil.

\footnotetext{
' Dep. Entomologia, Fitopatologia e Zoologia Agrícola. ESALQ/USP, Piracicaba, SP

2 Instituto Biológico. Campinas, SP.

${ }^{3}$ California Academy of Sciences. São Francisco, EUA.

${ }^{4}$ CSIRO. Canberra, Austrália.
} 